\title{
Editorial
}

\section{Proteomics in diagnostics}

\author{
Timothy D. Veenstra ${ }^{\mathrm{a}, *}$ and Sudhir Srivastava ${ }^{\mathrm{b}}$ \\ ${ }^{a}$ Laboratory of Proteomics and Analytical Technologies, SAIC-Frederick, Inc., National Cancer Institute at \\ Frederick, P.O. Box B, Frederick, MD 21702, USA \\ ${ }^{\mathrm{b}}$ Cancer Biomarkers Research Group, Division of Cancer Prevention, National Cancer Institute, Bethesda, MD \\ 20892, USA
}

Over the history of medicine, man has developed effective ways to treat many diseases that he has been presented with. The treatments range from prevention in the form of vaccination to the use of medicines and surgical intervention as cures or even treatments to control the diseases effect on the patients' quality of life. For many diseases, such as heart disease and cancer, early detection plays a pivotal role in the survival rate of the patient. When detected early, many such lethal diseases can be effectively treated with existing remedies. The difficulty remains, however, how to effectively detect such conditions at the earliest possible stage with a high enough positive predictive value so that they can be treated effectively without overwhelming the medical system with false positive diagnoses. While there are effective markers for diseases such as prostate (i.e. prostate-specific antigen) and ovarian cancer (i.e. cancer-antigen 125), these markers do not possess the sensitivity and specificity necessary to be used as a general screening tool in the diagnosis of early stage prostate and ovarian cancer. What is required is the identification of more effective or additional biomarkers, as well as other types of technologies, that can aid in the diagnosis of early stage diseases.

The challenge is how to identify more effective biomarkers or technologies that can provide an earlier

${ }^{*}$ Corresponding author: Dr. Timothy D. Veenstra, Biomedical Proteomics Program, SAIC-Frederick Inc., National Cancer Institute at Frederick, P.O. Box B, Frederick, MD 21702-1201, USA. Tel.: +1 301846 7286; Fax: +1 301846 6037; E-mail: veenstra@ncifcrf.gov. indication of a disease with a higher positive predictive value than presently utilized methods. Fortunately, the past decade has been witness to a new revolution in biological science. In this revolution, investigators now have the opportunity to study thousands of different biological molecules in a single experiment or in a series of high-throughput studies. This rate of characterization allows for the rapid comparison of samples from many different patients with the hope of finding species that are directly related to the disease state.

Proteomics, along with genomics and transcriptomics, has benefited greatly from the development of high-throughput methods to study thousands of proteins almost simultaneously. Inter-person heterogeneity is a major hurdle when attempting to discover a disease-related biomarker within biofluids such as serum, however, the coupling of high-throughput technologies and protein science now enables samples from hundreds of patients to be rapidly compared. Admittedly, proteomic approaches cannot remove the "finding a needle in a haystack" requirement for discovering novel biomarkers, however, we now posses the capability to inventory components within the "haystack" at an unprecedented rate. Indeed, such capabilities have already begun to bear fruit as our knowledge of the different types of proteins within serum is growing exponentially and novel technologies for diagnosing cancers using proteomic technologies are beginning to emerge.

Is the development of methods capable of characterizing thousands of proteins in a high-throughput manner going to identify novel biomarkers for the diagno- 
sis of early stage diseases or is the amount of data that is accumulated in such studies going to be too overwhelming? Based on the rate of interesting leads already being discovered using proteomics, it is likely that not only will biomarkers with better sensitivity and specificity be identified but individuals will be treated using customized therapies based on their specific protein profile. Since many of the proteomic technologies and data management tools are still in their infancy, the future of proteomics in disease diagnostics looks extremely promising. 


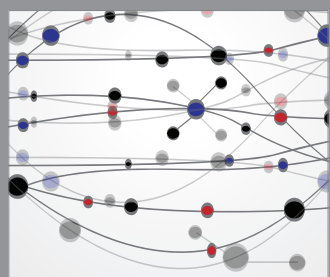

The Scientific World Journal
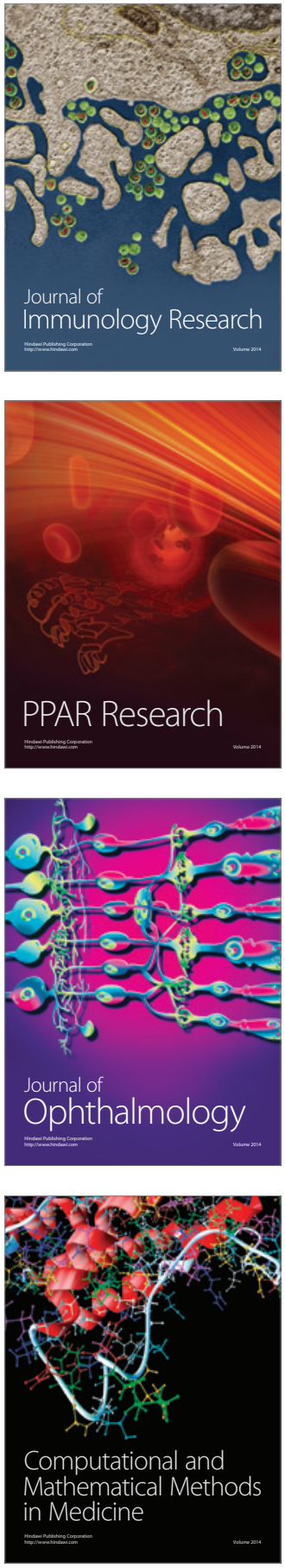

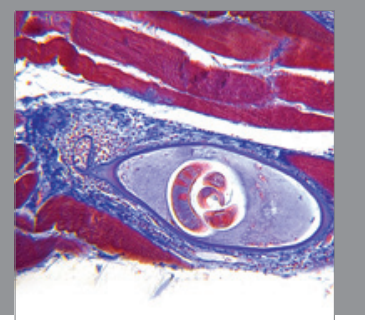

Gastroenterology

Research and Practice
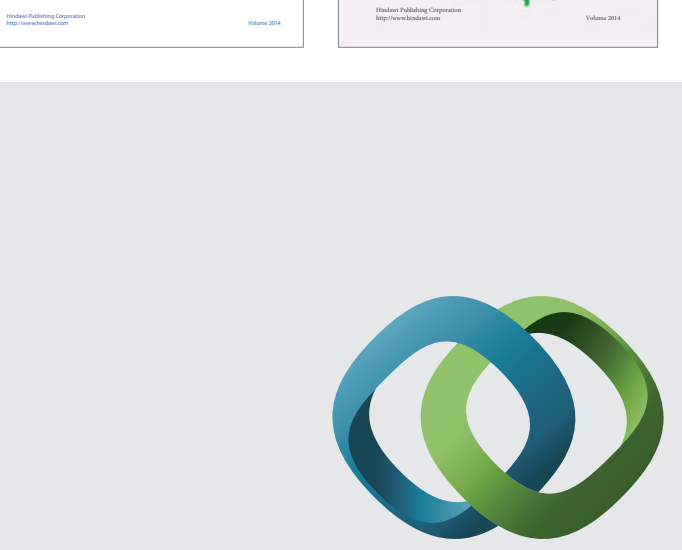

\section{Hindawi}

Submit your manuscripts at

http://www.hindawi.com
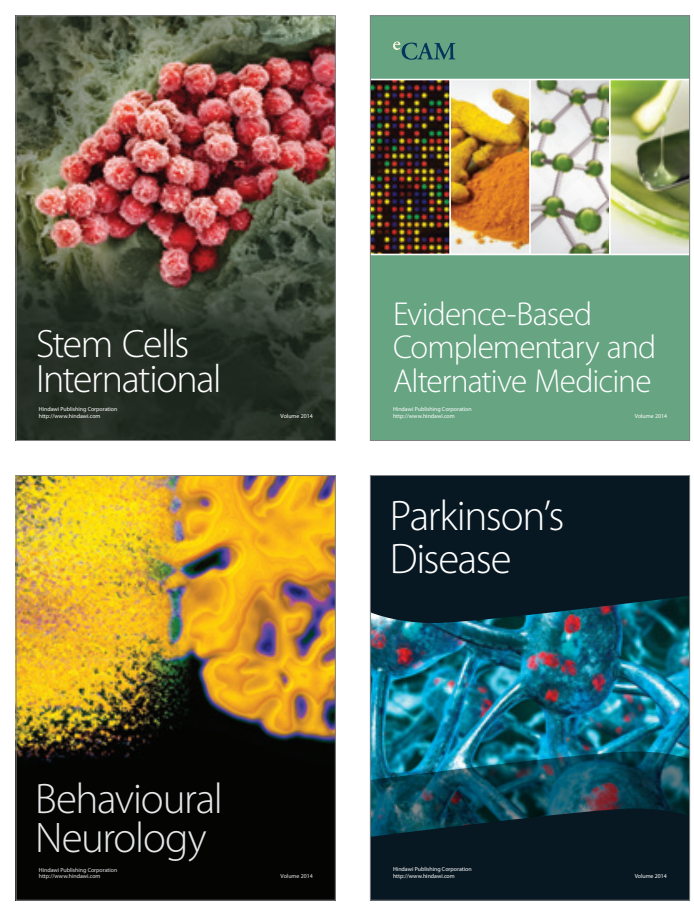

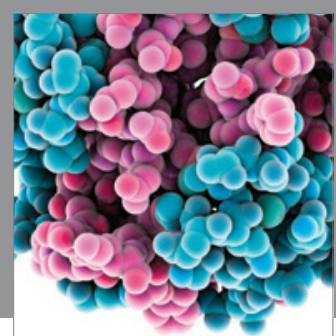

Journal of
Diabetes Research

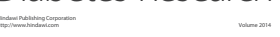

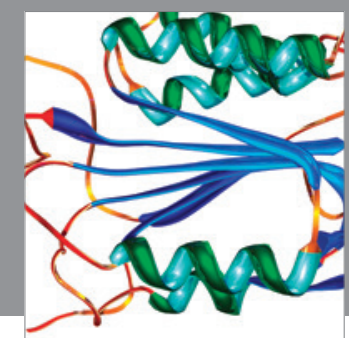

Disease Markers
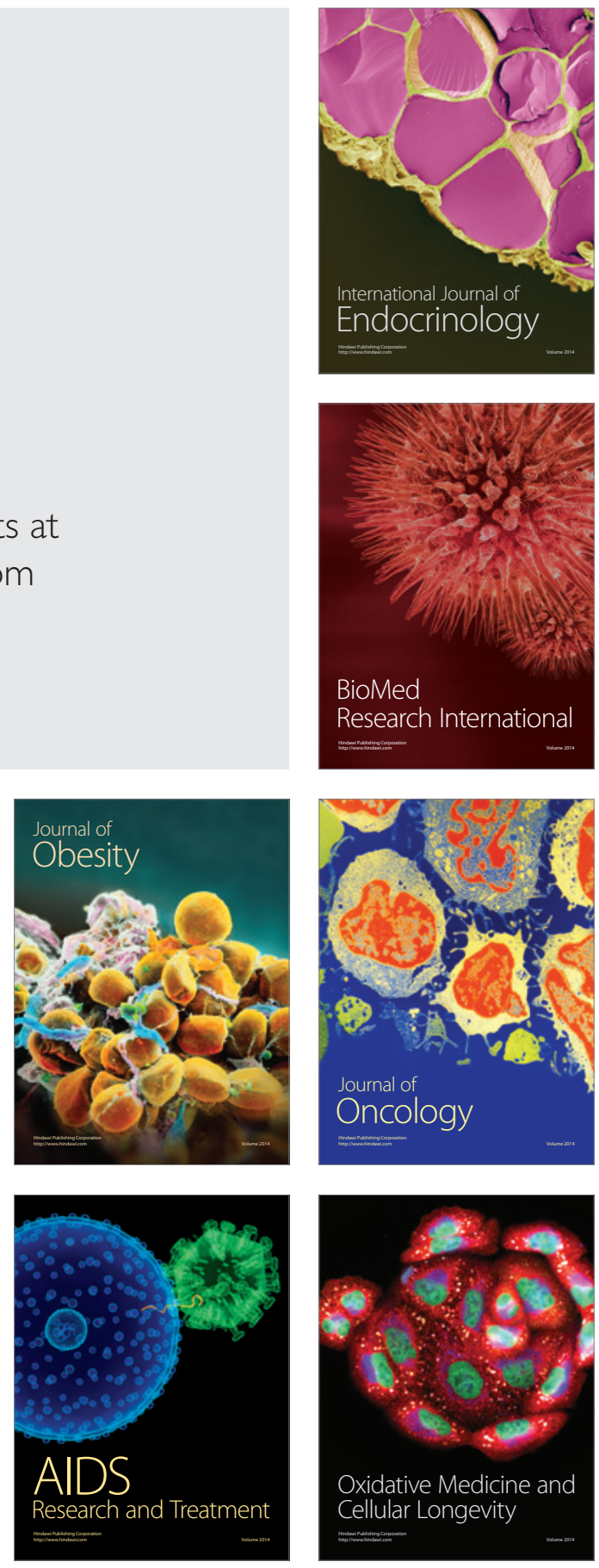